\title{
Risk assessment to improve patient care following cardioverter-defibrillator implantation
}

A straightforward risk score comprising ten readily-available preoperative variables that can be applied to patients preparing for cardioverter-defibrillator implantation has been developed by researchers. "We should be able to risk stratify patients better based upon preprocedural clinical variables and, in turn, justify the higher level of care for those that needed it," comments Dr David Haines, lead author of the article published in Circulation.

Data from 268,701 patients undergoing cardioverter-defibrillator implantation at 1,300 hospitals in the USA were collected in the ICD Registry between January 2006 and June 2008. A total of 8,589 patients (3.20\%) experienced acute, procedure-related complications, of whom 1,021 (0.38\%) died in hospital. The investigators shortlisted 29 potential variables recorded in the registry, and identified the following factors as the best predictors of complications following cardioverter-defibrillator

\author{
4 Patients with a score of $\geq 19$ \\ points ... should be identified for \\ higher-intensity care 77
}

implantation: age $\geq 70$ years ( 1 point), female sex ( 2 points), previous valvular surgery ( 3 points), device reimplantation other than for battery change (6 points), atrial fibrillation (1 point), NYHA class III or IV ( 1 or 3 points, respectively), chronic lung disease ( 2 points), blood urea nitrogen $>30 \mathrm{mg} / \mathrm{dl}$ (2 points), device type (dual chamber 2 points, or biventricular 4 points), and nonelective cardioverter-defibrillator implantation (3 points).

Patients with a score of $\geq 19$ points (3.9\% of the population) had a risk of in-hospital complications of $8.4 \%$, and should be identified for higher-intensity care following implantation. Indeed, the high risk of implantation might outweigh the long-term benefits of the device in some cases. On the other hand, patients scoring $\leq 5$ points ( $8.4 \%$ of the population) had a $0.6 \%$ complication risk and "should be able to go home the same day, thereby saving money and increasing patient convenience and satisfaction," hopes Dr Haines.

A 7-variable, 15-point score was similarly developed for predicting in-hospital death. The investigators are about to implement an algorithm for assigning patients scheduled to receive a cardioverter-defibrillator at Beaumont Hospitals, MI, USA to either same-day discharge or hospital admission on the basis of their scoring system.

Prospective follow-up will determine cost savings and patient outcomes.

Gregory B. Lim

Original article Haines, D. E. et al. Implantable cardioverter-defibrillator registry risk score models for acute procedural complications or death after implantable cardioverter-defibrillator implantation. Circulation 123, 2069-2076 (2011) 